1. That ectopic pregnancy reaching nearly up to harm. For instance, J. C. Wilson has recently treated the third month of gestation existed in the left Fal. a series of 147 cases of typhoid fover at the German lopian tube.

2. That some time after the second month the tube ruptured into the corresponding broad ligament, forming an intraligamentous hematocele.

3. That the hematocele continued to increase until it also ruptured into the general peritoneal cavity, where the hemorrhage became partially encysted, forming a large intraperitoneal hematocele.

4. Coincidently or prior to the ectopic pregnancy an intraligamentous cyst formed in the opposite broad ligament.

5. The value of intravenous saline infusion was demonstrated in tiding over the dangerous crisis caused by shock and hemorrhage during the laparotomy required to extirpate the sac, evacuate the blood collection and secure the vessels.

6. Septic bowel infection following the intestinal paresis of a moderate (limited) postoperative peritonitis.

7. Septic infarct of liver through portal vein as a consequence.

8. Aspiration of liver and removal of typic streptococcal pus.

9. Free hepatotomy after resection of ribs, followed by final and complete recovery.

\title{
NAPHTHALIN IN TYPHOID FEVER.
}

A REMEDI IN PREVENTING INTESTINAL PUTREFACTION AND TYMPANITES.

BY ALBERT WOLDERT, Ph.G., M.D.

Assistant Physician Out-Patient Department of Howard Hospital; Physician to Out-Patient Department for Diseases of Children, St. Joseph's Hospital. PHILADELPHIA, PA.

At the present time, when the cold-bath treatment of typhoid fever has proven to be of such inestimable value in reducing the death-rate of this scourge of humanity from that of former days, it seems almost unnecessary to speak of any other agent than cold water in controlling the clinical manifestations of enteric fever. Since we have become able for the most part to hold in check the chief disturbing factors, such as fever, nervous exhaustion, and overpowering of the circulatory system by this medium, it is customary to lay but little stress upon any other agent in restoring health to those who suffer from this disease.

If one considers the most important symptoms or complications which require remedial measures, besides those above enumerated,it may be stated that they are meteorism or tympanites, diarrhea, hemorrhage from the bowel and peritonitis. A small excess of gas in the bowel, over that of the normal, is necessarily an accompaniment of a disease located for the most part in this region, and existing to such an extent in this disorder that in a series of 112 cases of typhoid fever at the Hospital of the University of Pennsylvania, Tyson met with the condition in 85 per cent. of the number treated. Under ordinary circumstances slight tympanitic distension of the abdomen calls for no direct medication, usually depending upon undigested food which, upon being corrected, induces relief of the symptom.

No one can safely deny at the present time that the tendency is to give few drugs in cases of typhoid fever, nor can it be denied that overdrugging does Hospital, Philadelphia, by the cold-bath treatment, a liquid diet and fow drugs, with a mortality of 3.4 per cent. At the Hospital of the University of Pennsylvania, under the same plan of treatment, Tyson's mortality in a series of 112 cases was 4.5 per cent.; and at the St. Agnes Hospital. Stahl reports a death-rate of 2.8 per cent. out of 144 cases. In most of these cases the complications were few and required no medicinal agents for their relief, yet it would be unfair to cast aside the records of other hospitals in which the mortality was greater than the figures above quoted. Taking the city as a whole, the mortality for the past five months has reached a fraction above 9 per cent.

In private practice the facilities found in well. equipped hospitals can not always be had, and tym. panites with diarrhea is a not uncommon occurrence. In many instances there may be found deep ulcerations in the bowel, and any kind of food may undergo fermentation and putrefaction, giving rise to distension of the parts to such a degree that diarrhea, hemorrhage from the bowel, or perforation may ensue. If excessive tympanites exists, not only are these dangers imminent, but the respiratory and circulatory functions may also become embarrassed.

It has been more than once demonstrated that it is absolutely impossible to sterilize the intestinal tract in the human species, and it must be admitted that at the present time there is no ideal intestinal antiseptic. Those which have been used with more or less favor are well known. The trouble with a great many of them is that they are too soluble and, being so, become absorbed or chemically changed long before they reach the focus of disease. If we had a reliable intestinal antiseptic that would limit the number of pathogenic micro-organisms in the smaller intestines, the natural defenders of the body-polynuclear leucocytes with their contained nuclein, fixed endothelial cells, the epithelial cells, the mucous membranes, glandular secretions, the power of neutralization and a certain amount of reserve force-would, no doubt, in the majority of instances, decide favorably the combat against those few micro-organisms which have already gained entrance into the circulatory system.

In order to eliminate all the fallacies which might occur in presenting ovidence regarding the beneficial treatment of the disease, Stengel has quoted from a statistician who stated that from a mathematic calculation a series of 8000 cases should be presented before any irrefragable conclusion could be deduced.

While not extolling the virtues, nor decrying the evils, of other remedies that have been given for the purpose of limiting the amount of putrefaction in the intestinal tract during the course of typhoid fever, I desire to report that in eight unselected cases of this disease treated during different years, and to which cases naphthalin had been given, there was but slight distension of the bowel in any case, and hemorrhage occurred in none. In at least one case gaseous distension seemed to be relieved by the use of naphthalin. In all these cases fractional doses of calomel were given at the onset, and cold baths, when possible, every time the temperature rose above $102.4 \mathrm{~F}$. A liquid diet and use of the bedpan were of course strictly enjoined.

As a rule, naphthalin was begun within the first day, or from the time the patient was first seen, and was repeated one or more times throughout the course 
of the disease. It was given in powder or capsule, in doses of from 5 to 10 grains (according to age), and repeated every three hours if the patient was awake, day and night for two days. (In some cases it was continued for three days.) In the case of adults, doses less than 10 grains did not appear to do much good. In no case did the drug seem to act as a cardiac depressant, the pulse generally being somewhat slow, regular, and of good volume; nor did any troublesome complication ensue from its use. In the majority of instances, at the expiration of two days from its first administration, baths did not seem to be required as frequently as in many cases which $I$ have treated without the use of this remedy.

The fact that the temperature does not seem to be controlled by the drug is an indication that it does not act upon the thermogenic centers. Frequently gaseous eructations occurred, but it did not appear to disturb the digestive functions, and, judging from the condition of the tongue, it seemed rather to be improved under the administration of naphthalin. Vom. iting occurred twice in one of the cases, the first time doubtless being caused by taking the medicine with milk, the second time from taking the drug shortly after the milk had been given. One should be careful to select only the purest form of the drug, as the impure product causes burning at the neck of the bladder, supposed to be caused by naphthosulphurous acid (Wolff). In all cases the urine was turned quite dark in appearance after its administration, but the amount of the urine did not seem to be diminished. No symptoms of nephritis ever developed.

Naphthalin (naphthalinum, naphthalene) is described in the "United States Dispensutory" (seventeenth edition) as follows: "Naphthalin is a hydro. carbon having the chemic formula $\mathrm{C}_{11} \mathrm{H}_{s}$. It is a white, shining crystalline substance soluble in chloroform, carbon disulphid, ether. naphtha and the oils, but insoluble in water. A good test for the purity of naphthalin is to warm a little of it in a test-tube with sulphuric acid. As little as 1 per cent. impurity will be indicated by its imparting a pinkish tint to the sulphuric acid; the depth of the color indicates the degree of impurity." Its medical properties are given as follows: "Naphthalin is possessed of antiseptic properties, and is poisonous to most fungi and probably most insects. First employed by Rossbach of Jena, in intestinal catarrh, it has been largely used in all forms of intestinal inflammation and in typhoid fever."

In all the cases of typhoid fever and other diseases for which it has been given by others, I believe one death has been ascribed to the use of naphthalin. As a great many cases of typhoid fever need no medicine of any kind after the first few days, it seems that the drug is specially indicated at the onset of tympanites; when paralysis of the bowel occurs from overdisten. sion, it could do no good. Bouchard states that after its administration he has found in the urine a body for the most part composed of sulphur resulting from the combination of naphthol and sulphur, a naphthosulphurous acid, which may be classed as a naphthosulphite of soda. This could only have occurred by sulphur having been borrowed from the organism, i. e., by destroying albumin or nitrogenous matter. In all cases in which naphthalin is given in moderate-sized doses, its odor can be easily detected in the urine and feces. I paid particular attention to the condition of the urine in two cases of typhoid fever, throughout the course of the disease, for the purpose of ascertaining whether or not any irritation of the kidneys ensued from the use of the remedy, and the following facts were noted:

Case 1.-A. F., white, male, aged 9 years, had suffered from scarlet fever in 1893, measles a year later, and whooping.cough three years ago. The patient began to lose appetite and lounge about the house on November 15, and complained of headache, lose of appetite, nausea and chilly sensations. The bowels had moved once daily. There had been no epistaxis. On November 17 the cheeks became flushed, and the patient was first seen on November 18 , at which time the temperature was $103.6 \mathrm{~F}$. He was given calomel in fractional doses, and in addition 5 grains of naphthalin every three hours, the latter being continued until the evening of November 25 . On November 28 a specimen of blood from the lobe of the ear yielded a positive Widal reaction. November 29 , the bowels had moved several times by epsom salts November 25 and 26 , and by an enema on November 28 . The urine had been clear (?) for several days and the patient passed about 1000 c.c. of urine daily.

November 29 the urine was examined. The amount was about 1000 c.c., amber in color-slightly tinted darkish from naphthatin--reaction highly acid, specific gravity 1018 , no albumin, but there was a slight reddish zone at the point of contact with the nitric acid, and a moderate amount of indican. The nitroprussid test detected no indol. The sediment contained abundant urates, uric acid, granular débris, and a few leucocytes. There were no casts, nor red blood corpuscles, when examined by $1 / 12$ oil-immersion lens and No. 4 oc. (Leitz).

While the following method for the estimation of indol is not recommended as furnishing exact results, it was employed for the purpose of obtaining an index to the relative amount of this product. A bout 4 grams of a hard particle of the feces was selected and agitated thoroughly with about 24 c.c. of hot water and filtered, about 12 c.c. of filtrate resulting, having a straw color and characteristic fecal odor. Tha sodium nitroprussid test for indol proved unreliable, as the folluwing results show: Filtrate (as above prepared), 3 c.c.; solution sodium nitroprussid (2 grams to 4 c.c. of water), 0.3 c.c. ; solution of soda, 0.2 c.c.; acetic acid, 0.7 c.c. Upon the addition of the sodium nitroprussid to the filtrate a brownish tint was given to the fluid (probably due to the nitroprussid itself), and upon the addition of acetic acid it turned still darker in color, but did not turn violet nor blue. A specimen of normal feces similarly prenared failed to yield the violet or blue color, as did also a freshly orepared alcoholic filtrate from normal feces. The reaction was not tried with a distilled product.

November 30,5 grains of naphthalin were given at 5 P.M., and repeated three hours later, and on December 1 , and to the evening of December 2, 5 grains were given every three hours as far as possible. day and night. Thus 130 grains had been taken at the time the urine and feces were selected. The urine amounted to about $1000 \mathrm{c}$.c., was blackish in color, of the odor of naphthalin, highly acid, specific gravity 1020 , and showed indican greatiy decreased over that of November 29. There was no albumin, glucose nor acetone, and no casts.

The bowels had moved on December 1 , and were again moved by enema on December 3, a portion of which was obtained for examination. As the nitroprussid test without distillation had proven unsatisfactory. the following method was tried and gave approximate results. Af ter obtaining the filtrate from the feces, in the same manner as in the first method above, the following test was made: nitrous acid 0.7 c.c. : nitric acid (C.P.) 2 c.c. To 4 c.c. of the filtrate in a test tube there was added 0.5 c.c. of the acid solution. On adding two or three drops, quite slowly, a turbidity or cloudiness was produced; gradually adding the acid solution until 0.5 c.c had been used, the filtrate imwe. diately assumed a light reddish tint most marked at the bottom of the test-tube. On standing five or ten minutes the fluid assumed a deeper reddish color, which I notice at the end of three months has remained permanent. At the end of twentyfour hours a faint blue or grayish sediment was deposited.

The following test also gave the color reaction for indol. A filtrate was prepared from feces in the same manner as above outlined, and to 4 c.c. of the warmed filtrate were added 0.7 c.c. dilute sulphuric acid and 1.4 c.c. of the following solution: amyl nitrite (one glass bead containing 0.3 c.c.); alcohol 30 c.c. On adding the nitrite solution a light reddish color was immediately produced, but became more brownish on standing five or ten minutes. A filtrate produced with alcohol yielded a darker or mahogany red color with the amyl nitrite test. Comparing the results with that obtained from an examination of normal feces made at the same time, there seemed to be a lessened amount of indol in the stool from the typhoid patient. However, it must be added that in another comparative 
test made in the same manner, the results did not differ the one from the other, so that it does not always follow that indol is diminished in the feces after the administration of naph thalin.

In order to show the effects of naphthalin on urine from day to day, as well as to study the effects produced by its adminis tration during an attack of diarrhea occurring during the course of typhoid fever, reference to the following case may be interesting. (A due allowance must be made for paregoric and bis muth subnitrate in controlling the diarrhea while the naphthalin was being administered, and it is admitted that many fallacies are possible from the intermixture of effects of the drugs.)

Case 2.-W. H., male, American, aged 17 years, had suffered from pneumonia eight years previously; was otherwise healthy until the onset of this attack. He had been feeling unwell for several days previous to Saturday, December 31, but on that day symptoms gradually increased in severity, mani. fested by headache, which continued for several days. The bowels had moved once daily. On January 3 the patient began to suffer from headache again accompanied by pain in the back and limbs. At this time slight fever was present and the bowels became loose, moving two or three times during the day, and the patient ceased work on January 4. The bowels continued to be loose, with from two to three stools daily. Epistaxis and pains in the abdominal region came on, and on January 5 he suffered from nosebleeding again, with bowels still loose. The patient's mother had noticed that for the previous several days his face had become flushed toward the afternoon and it was thought there had been fever present.

The patient was first seen on January 8 , and the following symptoms noted: The abdomen was fiat, with no pain on pressure. The pupils were normal, facial expression dull and list less, tongue moist and slightly coated, whitish in certain areas. A faint systolic murmur was heard in the fifth interspace, corresponding to the apex-beat, and sibilant râles anteriorly. The pulse was full and strong. He was given fractional doses of calomel, which acted four times during the day. January 9 , a specimen of blood obtained from the lobe of the ear gave the positive Widal reaction. January 10 , the patient complained of having been restless during the previous night, remaining awaks until 4 A.M. ; suffered from considerable pain in abdom. inal region; tongue becoming heavily coated, the edges being normal in color; patient appeared very dull and was hardly able to retain the thermometer between the teeth. The spleen did not seem to be enlarged. The peripheral circulation appeared sluggish. The urine, on January 10, was dark, amber color, odor normal, sp.gr. 1022, acid reaction, no albumin, nogiucose, and not a trace of indican; urea was 1 per cent. and taking $1200 \mathrm{c} . \mathrm{c}$ as the amount passed in twenty-four hours, equaled 11 grams (164 grains) in the daily output. The sediment was composed of urates, uric acid crystals and granular débris. There were no casts. The patient had not taken any medicine of any kind since the calomel purge two days previously, $\theta x$. cept .33 grams of phenacetin on that day - the temperature being subsequently controlled by cold baths. A milk diet and use of the bedpan were strictly enjoined and upright posture during the taking of nourishment was forbidden. The pulse was full and strong, 86 per minute; bowels had moved five or eix times during the day, but there had been no blood in the feces, $\mathrm{He}$ had taken about sixty.four ounces of milk and possibly sixteen ounces of strained soup during the day.

On account of the diarrhea, $8 \mathrm{c}$.c. of camphorated tincture of opium was advised every three hours, beginning at $7: 30 \mathrm{P} . \mathrm{M}$.

January 10: The patient had slept about six hours during the night; bowels had not acted since the previous day; spleen slightly enlarged, but not tender; toward afternoon the bowels became loose, moving four times, and camphorated tincture of opium (8 c.c.) with bismuth subnitrate $(0.8$ grams) was given every three hours until four doses had been taken.

At 11 A.M. he began to take naphthalin, 0.7 grams every three hours day and night, the paregoric and bismuth also being continued.

January 12: The patient did not sleep well during the night and complained of colicky pains in the lower abdominal and lumbar regions. The bowels acted at 3 and 5 A.M. Paregoric, bismuth and naphthalin were continued.

On inquiry it was learned that the milk had been given when quite cold, for which warmer milk was substituted, containing a small amount of lime-water. The urine, which had been amber colored until January 11 , at 8 P.M., began to turn dark from the naphthalin. It was examined and showed a specific gravity of 1026 , and indican still absent-in order to confirm the absence of indican both sodium hypochlorite and calcium hypo chlorite were used. The urine had the odor of naphthalin and w as very dark in color. A slight burning pain was complained of in the region of the neck of the bladder. The pulse was reg. ular and full. In one stool particles of undigested milk were found. Owing to neglect, sordes had been allowed to collect about the teeth and gums, but which were subsequently kept clean by means of a weak solution of glycerin and persistent use of a tooth-brush.

January 13: The patient slept about five hours during the night, entirely free from pain, except slight pain on micturition. After 3 A.M. the bowels had moved three times. At 10 A.M. the pulse was 60 , regular and full, and the temperature registered 97.8 degrees. At 6 P.M. the pulse was 80 ; temperature 103.4. Naph thalin was continued until January 22 , the patient having taken 160 grains since January 10 . The bowels did not move any more during the day, subsequent to the morning action, and the paregoric and bismuth were discontinued. Boiled milk was now given, after which there was no more trouble with the bowels throughout the course of disease.

January 14: At 6 P.M. a bath was given, it being the only one necessary during the day. The mother stated that the patient had passed a good day. Free perspiration had occurred in the morning hours. The tongue was moist and had begun to clear off about the edges; bowels moved once. At 5 P.M. the urine was collected, was dark in color and contained no albumin nor indican. The urea was 0.9 per cent. and estimating that 1200 c.c. had been passed during the twenty-four hours, equaled 156 grains excreted during this period. One waxy cast was found, but a careful search failed to reveal any more. There was no blood. The heart murmur had now disappeared.

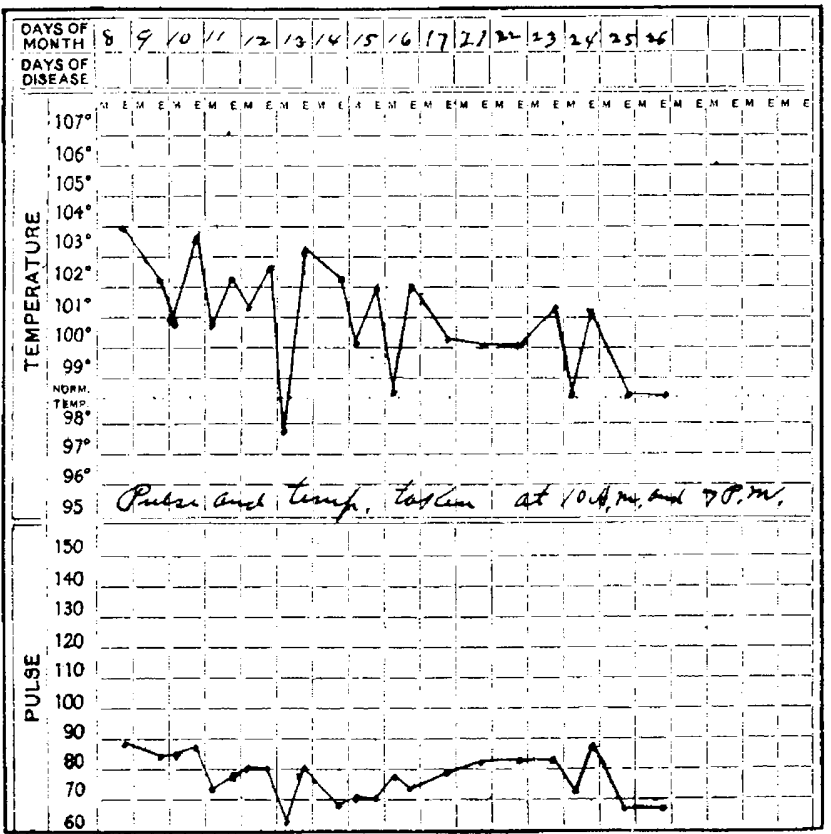

From January 22, naphthalin 0.7 grams (gr. $x$ ) was given every three hours, and the urine collected January 24, after 80 grains of naphtalin had been given. The results of examination were: Amount, 1200 c.c. ( 36 ounces); color blackish ; odor of naphtalin; sp. gr. 1015 ; acid reaction; no albumin ; no glucose. In the sediment were found a few crystals of uric acid and amorphous urates. There were no red blood-cells, no leucocytes, and no casts. The urea equaled 1 per cent. or 15 grams (164 grains) by one apparatus, and 9 grams (131 grains) by another, showing the variability of instruments used for the purpose of estimating this constituent of the urine.

From a study of these two cases it does not appear that naphtalin causes irritation of the kidneys.

None of these eight patients to which naphtalin had been given required any stimulant, such as alcohol, digitalis, or strychnia, throughout the course of the disease, and each made a good recovery.

NoTE.-The tests for indol were modified from von Jaksch. In making the test containing the nitrite of amyl, care must be exercised in breaking the grlass bead, ospecially if the graduate contains alcohol, for the nitrite is libera ted with such force as to
distance, thus endangering the eyesiont.

Dr. Joseph Thomas of Quakerstown, Pa., was recently elected president of the State Asylum for the Insane at Norristown. 